\title{
A METHODOLOGY FOR INVESTIGATING HEAT STRESS SELECTIVITY EFFECTS ON MENTAL PERFORMANCE
}

\author{
Ioannis Vasmatzidis \\ Robert E. Schlegel \\ University of Oklahoma \\ Norman, OK, 73019
}

\begin{abstract}
This paper outlines a methodology that can be used to investigate the selectivity patterns of heat stress effects. The adopted view is that heat stress causes performance to deteriorate because it depletes attentional resources. The term "selectivity" refers to the extent to which certain individual resource pools (the existence of which is postulated by multiple resource theories) are more susceptible to heat stress effects than others. The methodology consists of plotting performance of two time-shared tasks over time on the Performance Operating Characteristic (POC) space. Manipulating the difficulties of the paired tasks under the same environmental conditions (i.e., temperature level and exposure duration) produces a predictable change of the POC path. In particular, if the heat stress effects are non-selective, the POC path will rotate either clockwise or counter-clockwise depending on the task whose difficulty is increased. On the contrary, if the heat stress effects are selective, increasing the difficulty of a task will have no effect on the orientation of the POC path.
\end{abstract}

\section{INTRODUCTION}

The effects of heat stress on mental performance have been a traditional subject of inquiry for ergonomists and human factors specialists. As a result, a large number of studies have been conducted over the past decades to address the issue. However, from a theoretical standpoint little is known about the mechanisms of heat stress. The need for further development in this area has been identified in two recent review articles (Enander, 1989; Enander and Hugge, 1990). Theoretical knowledge gains importance with the emergence of information overload as one of the most significant problems in the design and use of complex industrial and military systems. In such environments, the minimization of human error is a crucial design priority. Consequently, the systems engineer and human factors engineer should be able to predict the impact of environmental stressors on the information processing capacity of the system operator.

This paper discusses the theoretical background of a proposed methodology for studying heat stress selectivity. The methodology assumes that degradation in the heat occurs due to attentional resource depletion as postulated by the maximal adaptability model (Hancock and Warm, 1989). The term "selectivity" refers to the extent to which certain individual resource pools (Wickens, 1987) are more susceptible to heat stress effects than others. Lack of a heat stress selectivity effect would indicate that heat drains attentional resources from a single, undifferentiated resource pool (Kahneman, 1973). The methodology is based on the Performance-Resource Function (PRF; Norman and Bobrow, 1975) and makes its inferences by recording on the Performance Operating Characteristic (POC) space the performance for two time-shared tasks performed in a hot environment. In particular, by plotting performance over time on the POC space under different task difficulty conditions, conclusions can be drawn regarding the heat selectivity pattern.

\section{THE MAXIMAL ADAPTABILITY MODEL}

In the past, arousal theory has been used almost exclusively by stress theorists to interpret heat stress experimental results. However, the theory has been invalidated at least in relation to heat stress research. Hancock (1987), for example, provided a set of convincing argunnents against the utility of arousal theory:

1. The theory is highly descriptive, but its predictive power is very limited. The well-known inverted-U relationship has been rarely quantified in the literature and in general it is freely moved to any location within the performance-arousal domain to fit any available data set.

2. The theory postulates a causal relationship between environmental stimulation, arousal and behavioral outcome. This temporal model, however, is not free of problems. For example, the theory implies that it is the absolute level of environmental stimulation that imposes its arousing (or dearousing) effect through the peripheral and central nervous systems. On the contrary, it seems that it is the magnitude of the environmental changes, and not the absolute level per se, that defines the level of arousal.

3. The nature of arousal as a unitary entity has been challenged. For example, Pribram and McCuiness (1975) proposed three energetical systems that control the functioning of attention: arousal, activation and effort.

A recent alternative to arousal theory is the maximal adaptability model developed by Hancock and Warm (1989). The model, illustrated in Figure 1, assumes that heat exerts its detrimental effects on performance by draining attentional resources. According to the model, input stress can vary from a negative extreme (hypostress) to a positive extreme (hyperstress). In the middle of this continuum is an area of minimal stress (normative zone) which requires no compensation on the part of the individual. Surrounding the normative zone is the comfort zone in which cognitive 


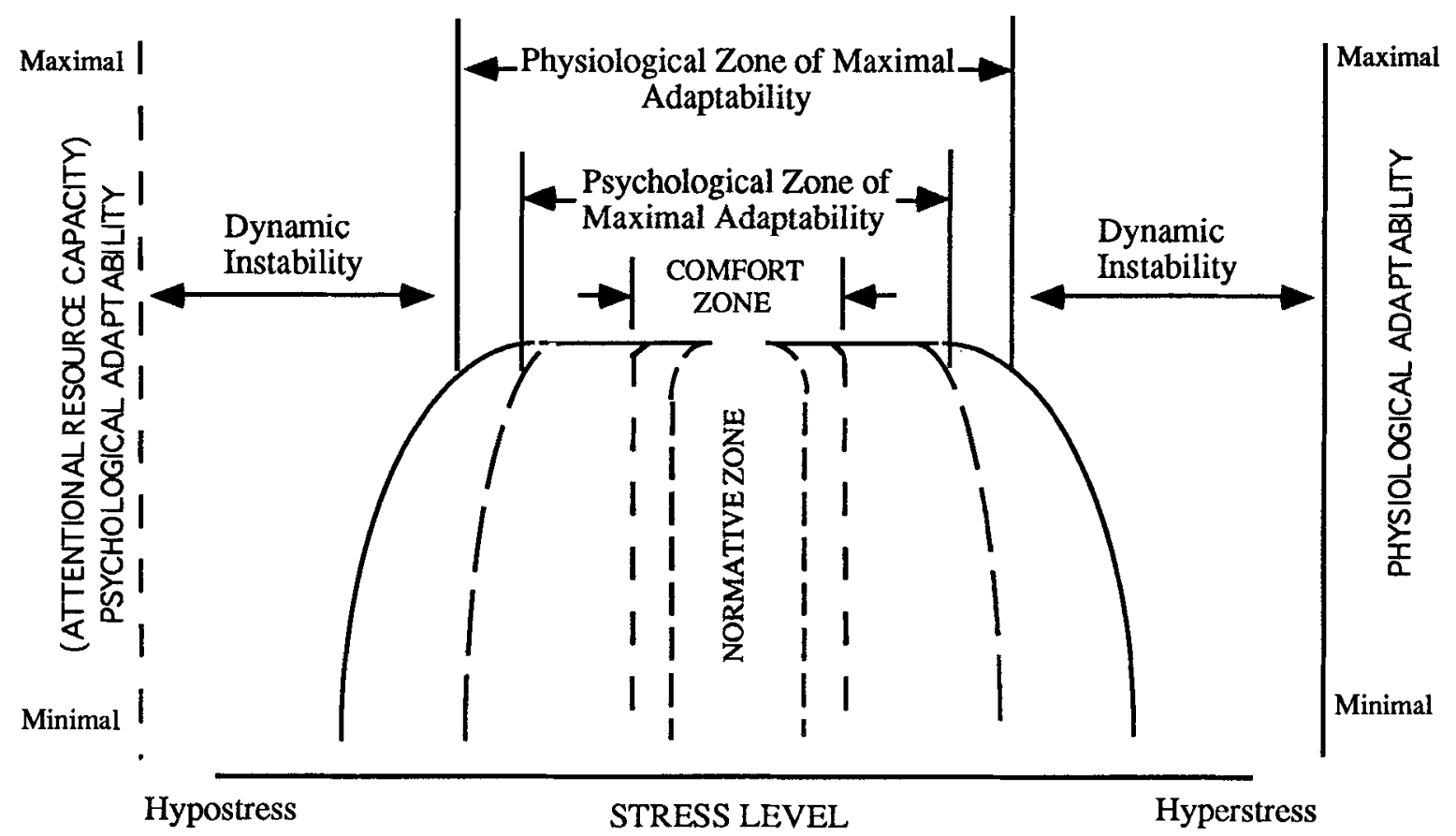

Figure 1. The Maximal Adaptability Model (Hancock and Warm, 1989)

adjustments to task demands are easily obtained and therefore performance is high. As the level of stress increases (by increasing exposure duration or intensity level of the stressor or both), attentional resources are progressively drained. Initially, the remaining resources are efficiently utilized by the individual with the net result being no performance decrement and sometimes performance improvement. This behavior is a reflection of psychological adaptability and is observed within the zone of maximal psychological adaptability in Figure 1. At higher levels of stress, the drain of attentional resources eventually damages performance. At these stress levels, performance deteriorates at an exponential rate. This is shown in Figure 1 by the steep fall of the dotted line representing the psychological zone of maximal adaptability. Further increases in stressor intensity move the body outside the zone of homeostasis (physiological zone of maximal adaptability) toward the region of dynamic instability, a life-threatening situation.

The above formulation assumes that resources are drained from a global, undifferentiated pool. This view is justified as long as no data are available to assume otherwise, a point also made by Hancock and Warm (1989; p. 527) The proposed methodology elaborates on this hypothesis by searching for possible heat stress selectivity patterns.

\section{THE METHODOLOGY}

Suppose that an individual is performing a dual-task paradigm in a hot environment. The Performance-Resource Functions (PRFs) of tasks $X$ and $Y$ are represented by the solid lines in Figures 2 and 3 respectively. Under conditions of equal task importance and assuming a single resource pool, $50 \%$ of the resources are invested in task X (point B in Figure 2 ) and the remaining $50 \%$ are invested in task $Y$ (point $D$ in Figure 3). On the corresponding POC space, this performance is represented by point $O$ in Figure 4. According to the concept of maximal adaptability, performance on the two tasks will eventually suffer due to attentional resource depletion. If heat stress effects are non-selective, the performance changes will be shown in Figures 2 and 3 by paths BC and DE respectively, which depict performance change over time in the tested hot environment. The resulting path on the POC space is OP which reflects a performance decrement $\triangle \mathrm{PX}_{\mathrm{X}}$ on task $\mathrm{X}$ equal to $\mathrm{P}_{\mathrm{B}}-\mathrm{P}_{\mathrm{C}}$, and a performance decrement $\Delta \mathrm{P}_{\mathrm{Y}}$ on task $\mathrm{Y}$ equal to $\mathrm{P}_{\mathrm{D}}-\mathrm{P}_{\mathrm{E}}$. Under the non-selectivity hypothesis, increasing the difficulty of either of the time-shared tasks will produce the following predicted pattern of performance change on the POC space. If, for example, the difficulty of task $X$ is increased (dashed PRF in Figure 2) while the difficulty of task $Y$ remains the same (solid PRF in Figure 3), and the individual is exposed to the same environmental conditions as when performing the easy version of task X (i.e., same temperature and exposure duration), the performance decrement for task $X$ will now be described by path $\mathrm{B}_{1} \mathrm{C}_{1}$ (Figure 2).

The performance decrement on task $Y$ is still shown by path $\mathrm{DE}$ (Figure 3). The new path on the POC space is now $\mathrm{O}_{1} \mathrm{P}_{1}$ (Figure 4a) and is characterized by a performance decrement $\triangle \mathrm{PX} 1$ on task $\mathrm{X}$ which is of smaller magnitude than $\Delta \mathrm{PX}_{\mathrm{X}}$. This follows from the fact that the total amount of resources depleted by a particular level of heat stress is the same for both task difficulty conditions.

Similarly, if a difficult version of task Y (dashed PRF in Figure 3 ) is paired with the easier version of task $X$ (solid PRF in Figure 2) under the same environmental conditions as before, the change in performance on both tasks is now represented by path $\mathrm{O}_{2} \mathrm{P}_{2}$ (Figure 4a) on the POC space. Path $\mathrm{O}_{2} \mathrm{P}_{2}$ reflects the same performance change on task $\mathrm{X}$ as $\mathrm{OP}$ but the performance decrement $\triangle \mathrm{PY} 1$ on task $\mathrm{Y}$ is less than $\Delta \mathrm{P}_{\mathrm{Y}}$ for the same reason as outlined earlier. By plotting the three POC paths of Figure 4a using a common origin (point $Q$ in Figure $4 \mathrm{~b}$ ), the effect of manipulating the task difficulties 
becomes obvious. In particular, increasing the difficulty of task X causes path QP (which is the same as OP) to rotate clockwise to $\mathrm{QP}_{1}$ (which is the same as $\mathrm{O}_{1} \mathrm{P}_{1}$ ), whereas increasing the difficulty of task $Y$ causes $Q P$ to rotate counterclockwise to $\mathrm{QP}_{2}$ (which is the same as $\mathrm{O}_{2} \mathrm{P}_{2}$ )

The spatial relationships illustrated in Figure $4 \mathrm{~b}$ will be obtained only if the effects of heat stress are non-selective. In the case of selectivity, manipulation of the difficulty of the task which is not sensitive to heat stress will not alter the POC path orientation. This behavior is illustrated in Figures 5,6 and 7 . Assuming that task $X$ is sensitive to the heat, but task $Y$ is not, time-sharing $X$ with $Y$ in a hot environment will cause performance on $X$ to decline from $P_{C}$ to $P_{D}$ whereas performance on $Y$ will not be affected and will remain constant

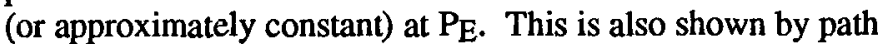
$O P$ in Figure 7a. If a more difficult version of task $Y$ (dashed line in Figure 7) is coupled with task $X$ under the same level of heat stress, the decline in performance for task $X$ will be the same as before (i.e., $\mathrm{P}_{C}-\mathrm{PD}_{\mathrm{D}}$ ) and performance on task $\mathrm{Y}$ will be $P_{E 1}$. This is also illustrated by path $O_{1} P_{1}$ in Figure 7a which is parallel to OP. Thus, if paths $O P$ and $O_{1} P_{1}$ are plotted with a common origin (see Figure $7 \mathrm{~b}$ ), no rotation of the initial path (OP) will be observed.

\section{DISCUSSION}

In summary, the described methodology consists of a POC plot of the performance of two time-shared tasks under different task difficulty conditions, while maintaining the same level of heat stress. In particular, the methodology predicts the following.

1. A clockwise or counter-clockwise rotation of the initial POC path will be obtained if heat stress effects are nonselective.

2. No rotation of the initial POC path will be obtained if heat stress effects are selective.

Implementation of the methodology in experimental studies necessitates a more detailed elaboration of a number of issues which are now discussed.

1. The Performance-Resource Functions of tasks $X$ and $Y$ in the methodology were considered resource limited (see Norman and Bobrow, 1975). The analysis becomes more complex, although the logic remains the same, when one or both tasks are data-limited. In practical applications, resourcelimited tasks can be constructed by making the tasks sufficiently difficult, unpredictable or of a continuous nature.

2. Increasing task difficulty causes a PerformanceResource Function to move toward the horizontal axis (see Figures 2,3 and 6). That is, raising the difficulty of a task lowers the marginal efficiency of resource allocation throughout the entire resource domain. This view has been supported by other investigators (Navon and Gopher, 1980).

3 . The methodology assumes a 50\%-50\% resource allocation policy for the paired tasks. However, the actual amount of resources allocated on each task is not critical and the 50\%-50\% policy was chosen only for the sake of simplicity. What is critical is to maintain a similar resource allocation on the two tasks regardless of difficulty level. Experimental control of resource allocation is a difficult matter since the postulation of resources is a theoretical concept, and thus resources cannot be measured directly. Using carefully designed subject instructions is an indirect approach for controlling resource allocation. Previous studies have reported that subjects can in general follow experimental instructions successfully (Gopher, 1980; Gopher and Brickner, 1982). An alternative approach is to provide continuous feedback representing desired levels of performance. This alternative, however, requires data collection prior to the actual experiment in order to construct a mapping between resources and performance levels. Undoubtedly, this approach requires experimental validation and at this point is only recommended as a possible alternative.

4. Implementation of the methodology is independent of the particular multiple resource model the researcher chooses to follow. Therefore, the selected tasks could reflect resources either from a two-hemisphere pool model (Folson and Friedman, 1988), or from a model advocating more than two resource pools (Wickens, 1987). In the latter case, an example of a dual-task scenario would be pairing an unstable tracking task which requires predominantly response resources, with a continuous mental arithmetic task which demands working memory, central processing resources. The fundamental assumption of the methodology is that heat drains attentional resources. Determination that the effect is selective provides evidence for the existence of some multiple resource model. The lack of a selective effect provides no evidence for or against a multiple resource model.

5. It is recognized that the skill level of the performer may limit the applicability of the methodology. For example, Hancock (1986) reported that heat stress effects on performance are minimal for very skilled individuals who have reached an automated level of processing. To avoid such situations due to overlearning, it is suggested at this early stage of methodology development that candidate subjects be selected from a pool of individuals possessing minimal, if any, prior experience with the experimental tasks.

In conclusion, this methodology offers an avenue for studying heat stress selectivity effects. As such, it can be used as a tool for filling the gap in the literature concerning heat stress effects on dual-task performance. This gap is largely due to the lack of a sound theory and a feasible methodology that can be used to guide future experimental work.

\section{REFERENCES}

Enander, A.E. (1989). Effects of thermal stress on human performance. Scandinavian Journal of Work, Environment and Health, 15, (supplement 1), 27-33.

Enander, A.E., and Hugge, S. (1990). Thermal stress and human performance. Scandinavian Journal of Work, Environment and Health, 16, (supplement 1), 44-50.

Gopher, D. (1980). On the training of time sharing skills: an attention view-point. In Proceedings of the Human Factors Society 24th Annual Meeting, (pp. 259-263), Santa Monica, CA: Human Factors and Ergonomics Society.

Gopher, D., and Brickner, M. (1982). Different difficulty manipulations interact differently with task emphasis: Evidence for multiple resources. Journal of Experimental Psychology: Human Perception and Performance, 8, 146157.

Hancock, P.A. (1986). The effect of skill on performance under an environmental stressor. Aviation, Space and 
Environmental Medicine, 57, 59-64.

Hancock, P.A. (1987). Arousal theory, stress and performance: Problems of incorporating energetic aspects of behavior into human-machine systems function. In L.S. Mark, J.S. Warm, and R.L. Huston (Eds.), Ergonomics and human factors: Recent research (pp. 170179). New York: Springer-Verlag.

Hancock, P.A., and Warm, J.S. (1989). A dynamic model of stress and sustained attention. Human Factors, 31, 519537.

Kahneman, D. (1973). Attention and effort. Englewood Cliffs, NJ: Prentice Hall.

Navon, D., and Gopher, D. (1980). Task difficulty, resources and dual-task performance. In R. Nickerson (Ed.),
Attention and performance VIII (pp. 297-315), NJ: Erlbaum.

Norman, D., and Bobrow, D.G. (1975). On data-limited and resource-limited processes. Cognitive Psychology, 7, 4464.

Polson, M.C., and Friedman, A. (1988). Task-sharing within and between hemispheres: A multiple resource approach. Human Factors, 30, 633 -643.

Pribram, K.H., and McGuiness, D. (1975). Arousal, activation and effort in the control of attention. Psychological Review, 82, 116-149.

Wickens, C.D. (1987). Attention. In P.A. Hancock (Ed.), Human factors psychology (pp. 29-80). North Holland: Elsevier Science.

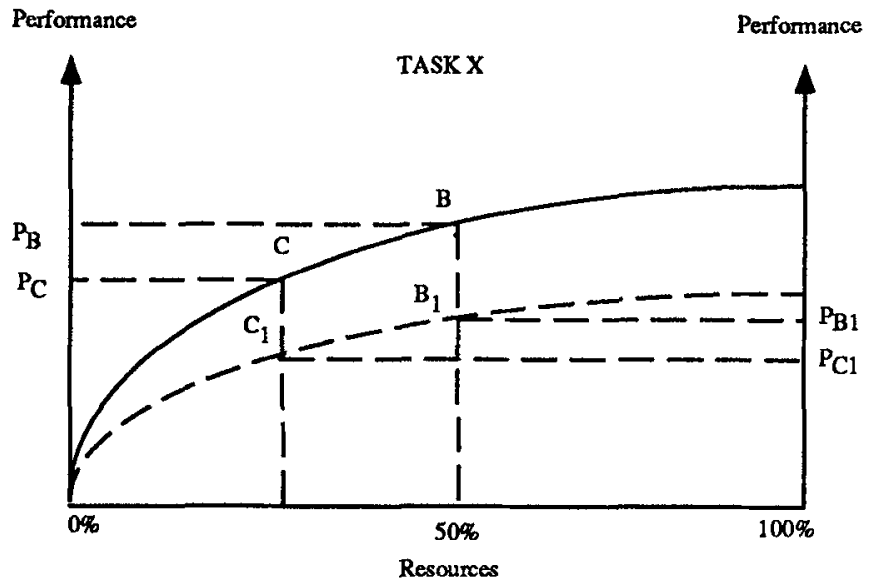

Figure 2. Performance-Resource Function for Task X.

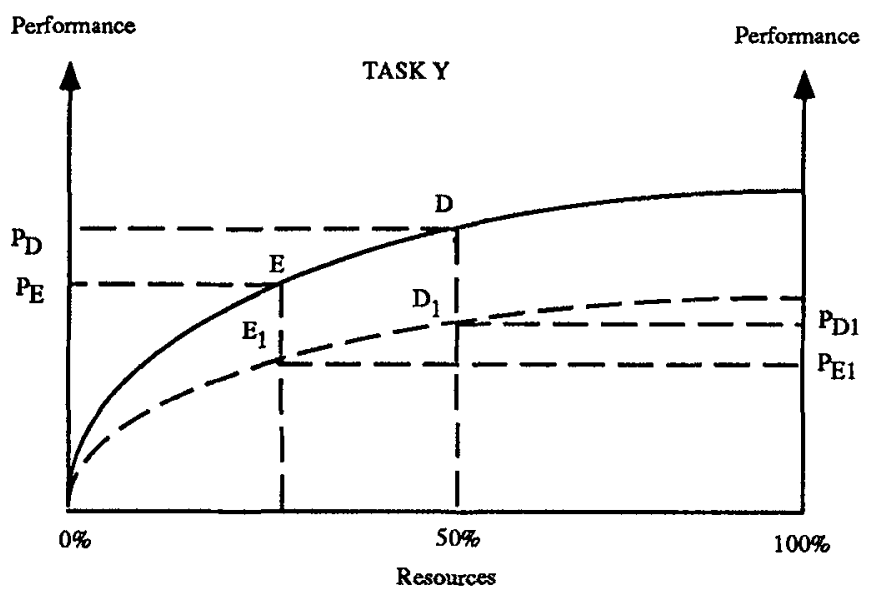

Figure 3. Performance-Resource Function for Task Y.

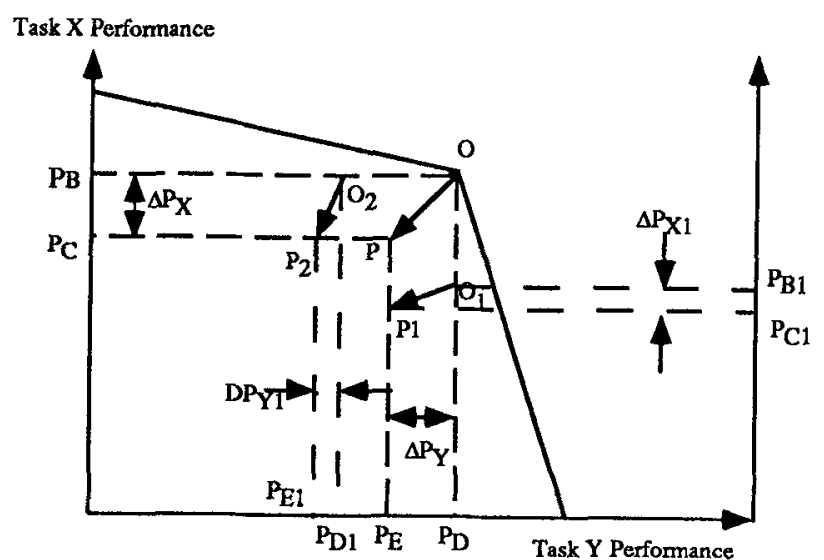

(a)

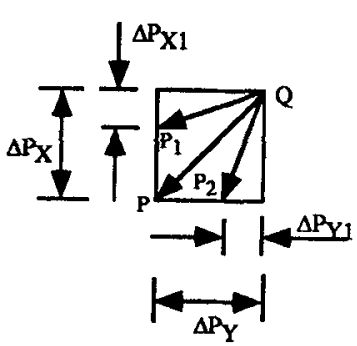

(b)

Figure 4. Effect of Task Difficulty Manipulation on Performance Operating Characteristic Paths when Heat Stress is Non-selective. 


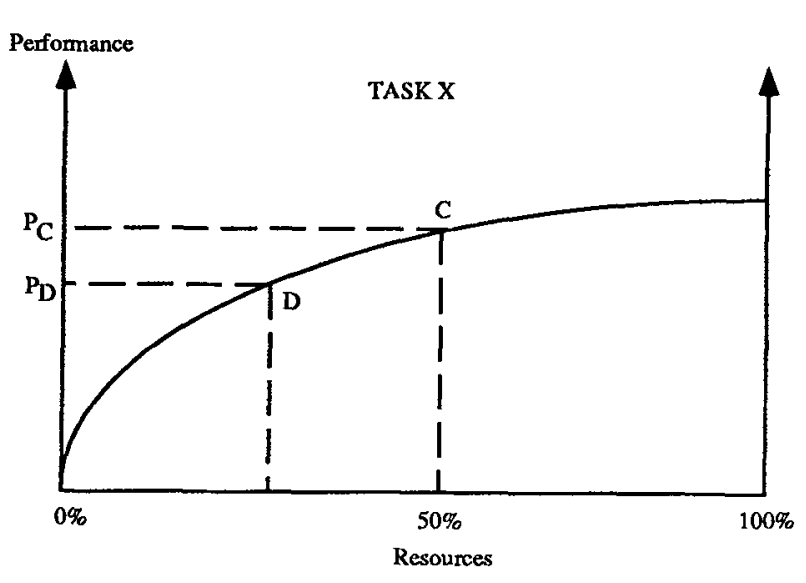

Figure 5. Performance-Resource Function for Task X.

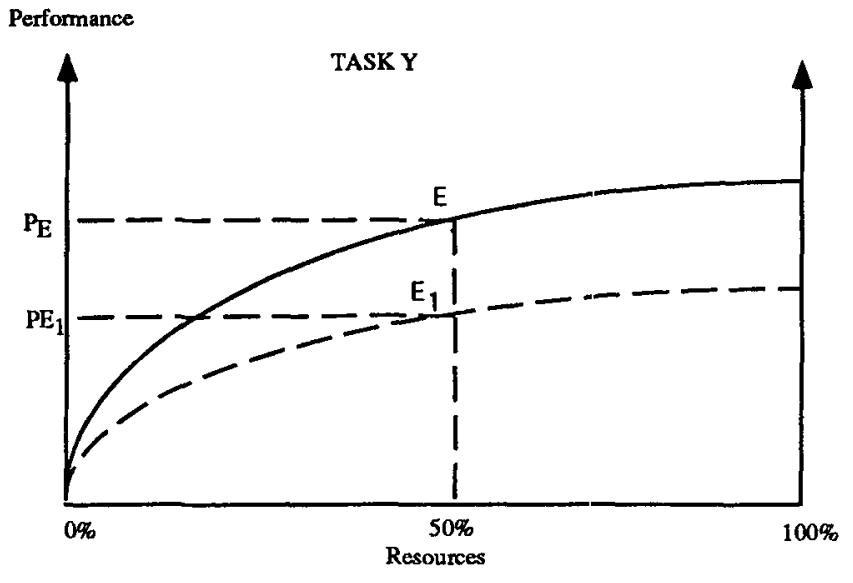

Figure 6. Performance-Resource Function for Task Y.
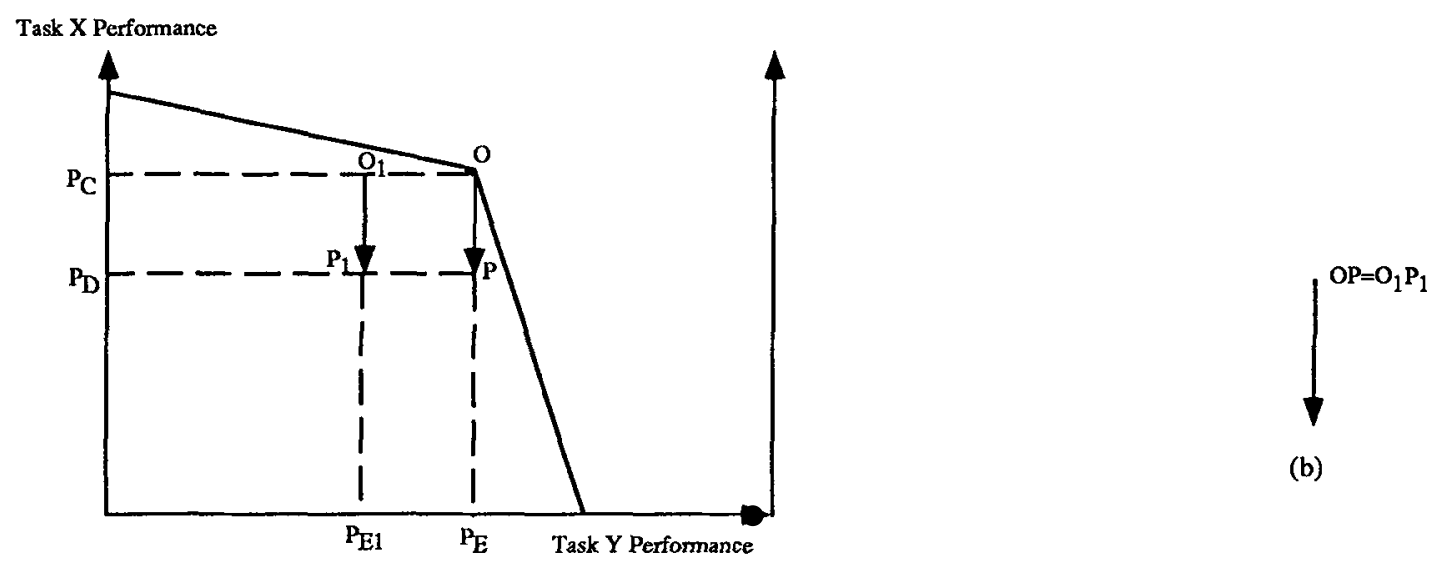

(b)

(a)

Figure 7. Effect of Task Difficulty Manipulation on Performance Operating Characteristic Path when Heat Stress is Selective. 